PROCEEDINGS OF THE

AMERICAN MATHEMATICAL SOCIETY

Volume 127, Number 6, Pages 1877-1883

S 0002-9939(99)04787-5

Article electronically published on February 18, 1999

\title{
THE TRANSVERSAL HOMOCLINIC POINTS ARE DENSE IN THE CODIMENSION-1 HÉNON-LIKE STRANGE ATTRACTORS
}

\author{
YONGLUO CAO \\ (Communicated by Mary Rees)
}

\begin{abstract}
We consider the codimension-1 Hénon-like strange attractors $\Lambda$. We prove that the transversal homoclinic points are dense in $\Lambda$, and that hyperbolic periodic points are dense in $\Lambda$. Moreover the hyperbolic periodic points that are heteroclinically related to the primary fixed point ( transversal intersection of stable and unstable manifolds) are dense in $\Lambda$.
\end{abstract}

\section{INTRODUCTION}

In 1976 [3] Hénon performed a numerical study of the family of diffeomorphism of the plane $h_{a, b}(x, y)=\left(1-a x^{2}+y, b x\right)$ and detected for parameter values $a=$ $1.4, b=0.3$, what seemed to be nontrivial attractor with a highly intricate geometric structure. This family has since then been the subject of intense research. But its dynamics are still far from being completely understood.

In a remarkable paper [1], Benedicks and Carleson show that if $b>0$ is small enough then for a positive measure set of $a$-values near $a=2$, the corresponding diffeomorphism $h_{a, b}$ exhibits a strange attractor.

Subsequently, this result has been much extended by Mora and Viana: The Hénon-like attractors appear whenever one generically unfolds a quadratic homoclinic tangency through one-parameter families of locally dissipative surface diffeomorphism [4].

Recently Viana [6] overcame many difficulties that arose when extending the BC method to a higher dimensional setting and showed the existence of codimension-1 Hénon-like strange attractors for unfoldings of codimension-1 homoclinic tangencies in higher dimensions.

In general in a manifold $M$ of dimension 2 or more, the codimension-1 Hénon-like strange attractors $\Lambda$ of diffeomorphism $\varphi$ is defined as follows:

1. There is a saddle fixed point $p \in \Lambda$ whose unstable manifold has dimension 1 , and $\Lambda$ is the closure of the unstable manifold of the saddle $p$.

2. The basin of $\Lambda$ contains an open set.

Received by the editors January 9, 1997 and, in revised form, September 30, 1997.

1991 Mathematics Subject Classification. Primary 58F11, 58F12.

Key words and phrases. Hénon-like strange attractor, transversal homoclinic point.

The author was partially supported by IMPA, TWAS and NSF of China and Jiangsu and "Nonlinear Science" Basic Project.

(C)1999 American Mathematical Society 
3. There is a point $z_{1}$ on the unstable manifold $w^{u}(p)$ such that its forward orbit is dense in $\Lambda$ and $\left\|\left(d \varphi^{n}\right)_{z_{1}}(\nu)\right\| \geq e^{c n}\|\nu\|$ for all $n \geq 0$, some constant $c>0$ and some nonzero vector $\nu$.

A construction of Sinai-Bowen-Ruelle (SRB) invariant measure for these attractors was given by Benedicks and Young [2].

There exists a direction in the tangent space at $z_{1}$ which is exponentially contracted by both positive and negative iterates of $D \varphi$, clearly the presence of such point is an obstruction to (uniform) hyperbolicity of the attractors. It implies the nonhyperbolicity of $\Lambda$.

It is well known that for an Axiom-A attractor, by the definition of hyperbolicity, it follows that the transversal homoclinic points are dense in it. Moreover the hyperbolic periodic points are dense in it, and any two periodic points are heteroclinically related (transversal intersection of stable and unstable manifolds).

We know that the codimension-1 Hénon-like strange attractors are nonhyperbolic. In this paper, we prove the following results.

Theorem. The transversal homoclinic points are dense in the codimension-1 Hénon-like strange attractor.

Corollary. The hyperbolic periodic points are dense in the codimension-1 Hénonlike strange attractor. Moreover the hyperbolic periodic points that are heteroclinically related to the primary fixed point also are dense in it.

This paper is arranged as follows. In Section 2 we give some preliminaries. Section 3 is devoted to the proof of the Theorem.

\section{Preliminaries}

In this section, we first give some definitions and facts.

First, we define the concept of a Hénon-like map, let $f_{a}(x)=1-a x^{2}$ and $\widehat{f}_{a}(x, Y)=\left(1-a x^{2}, 0^{m-1}\right)$, where $a \in[0,3],(x, Y) \in[-2,2] \times[-1,1]^{m-1}$.

Given $b>0$, a $C^{k}(k \geq 3)$ family of diffeomorphism $\varphi_{a}$ is a nonsingular $b$ perturbation of $\widehat{f}_{a}$, if $\|\varphi(a, x, Y)-\hat{f}(a, x, Y)\| \leq b$, where $\varphi(a, x, Y)=\varphi_{a}(x, Y)$, $\hat{f}(a, x, Y)=\widehat{f}_{a}(x, Y)=\left(1-a x^{2}, 0^{m-1}\right)$ and $\|\cdot\|$ is the $C^{k}$ norm.

It follows from the main Theorem in [1], Theorem B in [4] and the proof of Theorem $\mathrm{A}$ in [6] that if $b$ is small, then there exists a positive measure set $\Delta$ in the parameter space near 2 , the corresponding diffeomorphism $\varphi_{a}$ possesses a codimension-1 Hénon-like strange attractor $\Lambda$. Moreover $\Lambda=\overline{w^{u}\left(p_{a}\right)}$, where $p_{a}$ is the primary fixed point of $\varphi_{a}$, which is the continuation of the fixed point $\left(\frac{1}{2}, 0^{m-1}\right)$ of $\hat{f}_{2}$.

Now we recall some notations, concepts and definitions which will be used throughout the paper.

Fix $a \in \Delta$, and denote $\varphi=\varphi_{a}, \quad p=p_{a}$. Let $W$ denote the global unstable manifold of $p$.

2.1. The critical set. We use the following notation. For $A \in G L(m, R)$, if $v \mapsto$ $\frac{|A v|}{|v|}$ is not constant, let $f(A)$ denote the unit vector that is maximally expanding by $A$, and $e(A)=\{f(A)\}^{\perp}$ denote the contractive hyperplane of $A$. Write $e_{n}(z)=$ $e\left(D \varphi_{z}^{n}\right)$ if it makes sense. For $z \in W$, let $\tau \in T_{z} R^{m}$ denote a unit vector, tangent to $W$. 
A certain subset $\mathcal{C}$ of $W$ called the critical set is singled out in sects.5 and 6 of [1] and section 5 of [4] [6] to play the role of 0 in 1-dimension. Each $z_{0} \in \mathcal{C}$ has the property that tangent direction at $\varphi\left(z_{0}\right)$ is (exponentially) contracted by all forward iterates of $D \varphi$. This can be thought of as the moral equivalent to $f_{a}^{\prime}(0)=0$.

2.2. Controlled orbits. For $(v, V) \in R \times R^{m-1}$ we define $|\operatorname{slope}|(v, V)=\|V / v\|$.

Definition. A curve in $R^{m}$ is called a $C^{2}(b)$ curve if $\mid$ slope $\mid\left(\gamma^{\prime}\right) \leq 10 b$ and its curvature $\kappa \leq 10 b$.

(i) Let $z_{0}=\left(x_{0}, Y_{0}\right)$ be a critical point lying on a piece of $C^{2}(b)$ curve $\gamma \subset W$ and $\zeta_{0}=\left(\xi_{0}, \mathcal{Y}_{0}\right)$ be an arbitrary point in $(-\delta, \delta) \times R^{m-1}$. We say that $\zeta_{0}$ is in tangential position with respect to $z_{0}$ if there is $\widehat{z_{0}}=\left(\widehat{x_{0}}, \widehat{Y_{0}}\right) \in \gamma$ with $\widehat{x_{0}}=\xi_{0}$ and $\left|\widehat{Y_{0}}-\mathcal{Y}_{0}\right|<\left|\widehat{x_{0}}-x_{0}\right|^{4}$.

(ii) Let $\zeta_{0} \in W$, and let $\zeta_{n}=\varphi^{n} \zeta_{0}$. We say that the orbit of $\zeta_{0}$ is controlled on the time interval $[0, N), N \leq \infty$, if the following holds. If $\zeta_{0} \notin \mathcal{C}$, let $n_{1}<n_{2}<\cdots$ be the times when $\zeta_{n}$ is in $(-\delta, \delta) \times R^{m-1}$. If $\zeta_{0} \in \mathcal{C}$, take $n_{1}>0$ to be the first time $\zeta_{n}$ return to $(-\delta, \delta) \times R^{m-1}$. Then for every $n_{i} \in[0, N), \zeta_{n_{i}}$ is attached to a critical point with respect to which it is in tangential position. This critical point is denoted by $z\left(\zeta_{n_{i}}\right)$ and is called the binding point of $\zeta_{n_{i}}$.

2.3. Bound periods. Let $z_{0} \in \mathcal{C}$, and let $\zeta_{0}$ be in tangential position with respect to $z_{0}$. As a first approximation we define the bound period $\tilde{p}\left(\xi_{0}, z_{0}\right)$ to be the largest $k$ such that

$$
\left|\varphi^{j} \zeta_{0}-\varphi^{j} z_{0}\right|<e^{-\beta j} \quad j<k
$$

(Recalled that $\alpha=10^{-6}, \beta=14 \alpha$.)

Now consider $\zeta_{0}$ whose orbit is controlled on $[0, \infty)$, and let $\tilde{p}_{i}=\tilde{p}\left(\zeta_{n_{i}}, z\left(\zeta_{n_{i}}\right)\right)$. Note that it is entirely possible for $\zeta_{n}$ with $n_{i}<n<n_{i}+\tilde{p}_{i}$ to return to $(-\delta, \delta) \times$ $R^{m-1}$, so that bound periods can be initiated in the middle of bound periods. It is shown in section 6.2 of [1], section 8 of [4] and section 6 of [6] that by modifying slightly the definition of $\tilde{p}$, bound periods can be mode nested. More precisely, one can choose $\left\{p_{i}\right\}_{i=1}^{\infty}$ in such a way that for all $i$,

(i) $p_{i} \leq \tilde{p}_{i}$ and $\left|\varphi^{p_{i}} \zeta_{n_{i}}-\varphi^{p_{i}} z\left(\zeta_{n_{i}}\right)\right| \geq e^{-\beta^{*} p_{i}}$ for some $\beta^{*} \approx \beta$,

(ii) if $n_{j}$ is such that $n_{i}<n_{j}<n_{i}+p_{i}$, then $n_{j}+p_{j} \leq n_{i}+p_{i}$.

The bound period between $\zeta_{n_{i}}$ and $z\left(\zeta_{n_{i}}\right)$ is then defined to be $p_{i}$. It is further required that if the bound relation between $z_{n_{i}}$ and $z\left(z_{n_{i}}\right)$ is still in effect at time $n_{j}>n_{i}$, then we must have $z\left(z_{n_{j}}\right)=z\left(\varphi^{n_{j}-n_{i}} z\left(z_{n_{i}}\right)\right)$.

2.4. Bound of free states. Conceptually, it is convenient to divide a controlled orbit into free and bound states. Let $\zeta_{0}$ be as above.

- For $n \leq n_{1}, \zeta_{n}$ is free.

- For $n_{1}<n<n_{1}+p_{1}, \zeta_{n}$ is said to be in bound state.

- Let $n_{i}$ be the first return to $(-\delta, \delta) \times R^{m-1}$ after $n_{1}+p_{1}$. Then $\zeta_{n}$ is free for $n_{1}+p_{1} \leq n \leq n_{i}$, and $\zeta_{n_{i}}$ is called a free return.

- $\zeta_{n}$ is in bound state again during the period $n_{i}<n<n_{i}+p_{i}$, and so on.

Let $\mathcal{P}=\left\{I_{\mu_{j}}\right\}$ be the partition of $(-\delta, \delta)$, given by $(-\delta, \delta)=\bigcup_{|\mu| \geq \mu_{0}} I_{\mu}$, where $I_{\mu}=\left(e^{-(\mu+1)}, e^{-\mu}\right)$ for $\mu>0, \quad I_{\mu}=-I_{-\mu}$, for $\mu<0$. 
Lemma 2.1. Let $\xi_{0} \in W$ and assume that $\xi_{i} \notin \mathcal{C}$ for all $i$, then $\xi_{i}$ is in a free state infinitely often, and the following holds for $\tau_{i}=D \varphi_{\xi_{0}}^{i} \tau$, where $\tau$ denotes a unit vector, tangent to $W$ at point $\xi_{0}$.

(I) Expansion outside of $(-\delta, \delta) \times R^{m-1}$.

There are $c_{0}>0$ and $M_{0} \in Z^{+}$such that

(1) If $\xi_{i}$ is free and $\xi_{i}, \ldots \xi_{i+M_{0}} \notin(-\delta, \delta) \times R^{m-1}$, then

$$
\frac{\left|\tau_{i+M_{0}}\right|}{\left|\tau_{i}\right|} \geq e^{c_{0} M_{0}}
$$

(2) If $\xi_{i} \notin(-\delta, \delta) \times R^{m-1}$ is free, and $k>i$ is the first time return, namely $\xi_{k} \in(-\delta, \delta) \times R^{m-1}$, then

$$
\frac{\left|\tau_{k}\right|}{\left|\tau_{i}\right|} \geq e^{c_{0}(k-i)}
$$

(II) Estimate of bound period.

There is $c_{1} \approx \log 2$, such that following holds: If $\xi_{i} \in(-\delta, \delta) \times R^{m-1}$ is free and becomes bound at this time $z\left(\xi_{i}\right) \in \mathcal{C}$ with bound period $p$, then

(a) If $e^{-\mu-1} \leq\left|\xi_{i}-z\left(\xi_{i}\right)\right| \leq e^{-\mu}$, then $\frac{1}{2} \mu \leq p \leq 5 \mu$;

(b) $\frac{\left|\tau_{i+j}\right|}{\left|\tau_{i}\right|} \geq 3\left|\xi_{i}-z\left(\xi_{i}\right)\right| \cdot\left|D \varphi_{z\left(\xi_{i}\right)}^{j}\left(\begin{array}{c}0 \\ 1^{m-1}\end{array}\right)\right| \geq\left|\xi_{i}-z\left(\xi_{i}\right)\right| e^{c_{1} j}, 0 \leq j \leq p$;

(c) $\frac{\left|\tau_{i+p}\right|}{\left|\tau_{i}\right|} \geq e^{\frac{1}{3} c_{1} p}$.

Proof. See Corollary 1 in section 2.1.1 of [2].

For $x_{0}$ with $\left|x_{0}\right| \ll \delta$, let $\mathcal{P}_{\left[x_{0}\right]}$ denote a copy of $\mathcal{P}$ with 0 moved to $x_{0}$, more precisely, let $h:(-\delta, \delta) \rightarrow(-\delta, \delta)$ be a piecewise linear homeomorphism taking the points $-\delta,-e^{-\left(\mu_{0}+1\right)}, x_{0}, e^{-\left(\mu_{0}+1\right)}, \delta$, to $-\delta,-e^{-\left(\mu_{0}+1\right)}, 0, e^{-\left(\mu_{0}+1\right)}, \delta$ respectively and let $\mathcal{P}_{\left[x_{0}\right]}=h^{-1} \mathcal{P}$.

Furthermore, if $\gamma$ is a horizontal curve and $z_{0}=\left(x_{0}, Y_{0}\right)$ is such that $\left|x_{0}\right| \ll \delta$, then $\mathcal{P}_{\left[z_{0}\right]}=\mathcal{P}_{\left[x_{0}\right]}$ is a partition on $\gamma \cap\left((-\delta, \delta) \times R^{m-1}\right)$. Once $\gamma, z_{0}$ are fixed, for the sake of simplicity, we will consider $I_{\mu}$ as though it is a subsegment of $\gamma$. Let $J_{\mu}^{+}=I_{\mu-1} \cup I_{\mu} \cup I_{\mu+1}$, in particular, $J_{\mu_{0}}^{+}=I_{\mu_{0}-1} \cup I_{\mu_{0}} \cup I_{\mu_{0}+1}$.

Remark 1. For the point in $J_{\mu}^{+}$, we have defined bound period as in section 2.3, for a segment $I \subset J_{\mu}^{+}$, we define its bound period $p=\max _{x \in J_{\mu}^{+}} p(x)$, from Lemma 2.1, it follows that $\frac{\left|\tau_{p}\right|}{|\tau|} \geq e^{\frac{1}{6} c_{1} p}$.

If $\gamma_{0}$ is a curve segment, let $\gamma_{j}=\varphi^{j} \gamma_{0}$, when we say that a segment $\gamma \subset W$ is free, it will assumed implicitly that $\forall z \in \gamma, z$ is controlled during the time interval $(-\infty, 0)$.

Define the projection map $\pi: R \times R^{m-1} \rightarrow R$ by $\pi(x, Y)=x$.

Lemma 2.2. Let $\gamma$ be a free segment of $W$, then

(1) $\forall z \in \gamma, \quad \mid$ slope $\mid(\tau(z))<\frac{2 b}{\delta}$;

(2) $\forall z \in \gamma \cap(-\delta, \delta) \times R^{m-1}, \quad \mid$ slope $\mid(\tau(z))<10 b$.

Proof. See Lemma 1 in section 2.2.1 of [2].

\section{The MAIN THEOREM}

In this section, we will give the proof of the Theorem.

Lemma 3.1. For one-dimensional map $f_{2}(x)=1-2 x^{2}, \quad x \in[-1,1],\left\{f_{2}^{-n} \widehat{x}_{2}\right.$, $n \geq 0\}$ is dense on $[-1,1]$, where $\hat{x}_{2}$ is the fixed point of $f_{2}$. 
Proof. The map $f_{2}$ has an absolutely continuous invariant measure with positive entropy. Therefore $f_{2}$ is exact on $[-1,1]$, that is to say, given an interval $I \subset$ $[-1,1]$, there exists $N(I)$ such that $f_{2}^{N(I)} I=[-1,1]$, from which it follows that $\left\{f_{2}^{-n} \widehat{x}_{2}, n \geq 0\right\}$ is dense on $[-1,1]$.

Lemma 3.2. There exist $a_{0}, a_{1}$, sufficiently close to 2 with $a_{0}<a_{1}<2$, such that for the map $f_{a}, \quad a \in\left[a_{0}, a_{1}\right], \exists$ a neighbourhood $V$ of $\widehat{x}_{2}$ and intervals $B^{ \pm} \subset I_{ \pm \mu_{0}}$, $A^{ \pm} \subset I_{ \pm\left(\mu_{0}-1\right)}$ and two integers $N, M \in Z^{+}$such that $f_{a}^{N} B^{ \pm} \supset V, f_{a}^{M} A^{ \pm} \supset V$ and $\widehat{x}_{a} \in$ IntV, where $\widehat{x}_{a}$ is a fixed point of $f_{a}$ corresponding to $\widehat{x}_{2}$.

Proof. From Lemma 3.1, it follows that there exist intervals $B^{ \pm}$and $A^{ \pm}$, a neighbourhood $V$ of $\widehat{x}_{2}$ and $N, M \in Z^{+}$such that $f_{2}^{N} B^{ \pm} \supset V$ and $f_{2}^{M} A^{ \pm} \supset V$. By openness, this implies there are $a_{0}, a_{1}$ sufficiently near 2 , such that $\widehat{x}_{a} \in V$ and $f_{a}^{N} B^{ \pm} \supset V, f_{a}^{M} A^{ \pm} \supset V$ for $a \in\left[a_{0}, a_{1}\right]$.

Lemma 3.3. If $a \in\left[a_{0}, a_{1}\right]$, then both $\widehat{f}_{a}^{N}\left(B^{ \pm} \times\left\{0^{m-1}\right\}\right)$ and $\widehat{f}_{a}^{M}\left(A^{ \pm} \times\left\{0^{m-1}\right\}\right)$ intersect $w^{s}\left(\widehat{x}_{a}, 0^{m-1}\right)$ transversally.

Proof. For $\widehat{f}_{a}, a \in\left[a_{0}, a_{1}\right]$, the stable set of $\left(\widehat{x}_{a}, 0^{m-1}\right)$ contains a $(m-1)$-dimension hyperplane through the point $\left(\widehat{x}_{a}, 0^{m-1}\right)$, which is perpendicular to the $x$-axis. From Lemma 3.2 , it follows that $\widehat{f}_{a}^{N}\left(B^{ \pm} \times\left\{0^{m-1}\right\}\right)$ and $\widehat{f}_{a}^{M}\left(A^{ \pm} \times\left\{0^{m-1}\right\}\right)$ intersects $w^{s}\left(\widehat{x}_{a}, 0^{m-1}\right)$ transversally.

Lemma 3.4. There exists $b_{0}$ small enough such that if $b<b_{0}$ then both $\varphi_{a}^{N}\left(\tilde{B}^{ \pm}\right)$ and $\varphi_{a}^{M}\left(\tilde{A}^{ \pm}\right)$intersect $w^{s}\left(p_{a}\right)$, for $a \in\left[a_{0}, a_{1}\right]$, where $\tilde{B}^{ \pm} \subset B^{ \pm} \times[-1,1]^{m-1}$, $\tilde{A}^{ \pm} \subset A^{ \pm} \times[-1,1]^{m-1}$, and $\pi\left(\tilde{B}^{ \pm}\right)=B^{ \pm} \times\left\{0^{m-1}\right\}, \pi\left(\tilde{A}^{ \pm}\right)=A^{ \pm} \times\left\{0^{m-1}\right\}$, and $\tilde{B}^{ \pm}$(respectively $\tilde{A}^{ \pm}$) are close to $B^{ \pm} \times\left\{0^{m-1}\right\}$ (respectively $A^{ \pm} \times\left\{0^{m-1}\right\}$ ) in the sense of the Hausdorff metric.

Proof. According to the differentiable dependence of stable manifolds in a compact set and Lemma 3.3, it follows that there exists $b_{0}$ small enough that provided $b<b_{0}$, then for $a \in\left[a_{0}, a_{1}\right]$, both $\varphi_{a}^{N}\left(B^{ \pm} \times\left\{0^{m-1}\right\}\right)$ and $\varphi_{a}^{M}\left(A^{ \pm} \times\left\{0^{m-1}\right\}\right)$ intersect $w^{s}\left(p_{a}\right)$ transversally. Therefore if $\tilde{B}^{ \pm} \subset B^{ \pm} \times[-1,1]^{m-1}$ and $\pi\left(\tilde{B}^{ \pm}\right)=B^{ \pm} \times\left\{0^{m-1}\right\}, \quad \tilde{B}^{ \pm}$ is close to $B^{ \pm} \times\left\{0^{m-1}\right\}$ in the sense of the Hausdorff metric. Then because of the differentiable dependence on the map, $\varphi_{a}^{N}\left(\tilde{B}^{ \pm}\right)$intersects $w^{s}\left(p_{a}\right)$ transversally. Analogously, if $\tilde{A}^{ \pm} \subset A^{ \pm} \times[-1,1]^{m-1}$ and $\pi\left(\tilde{A}^{ \pm}\right)=A^{ \pm} \times\left\{0^{m-1}\right\}, \quad \tilde{A}^{ \pm}$is close to $A^{ \pm} \times\left\{0^{m-1}\right\}$ in the sense of the Hausdorff metric, then $\varphi_{a}^{M}\left(\tilde{A}^{ \pm}\right)$intersects $w^{s}\left(p_{a}\right)$ transversally.

Theorem. There exists $b_{0}>0$ small enough such that if $b<b_{0}$ then for $a \in$ $\Delta \cap\left[a_{0}, a_{1}\right]$ the transversal homoclinic points are dense in $\Lambda$.

Proof. Since $W$ is dense in $\Lambda$, it is sufficient to prove that the transversal homoclinic points are dense in $H_{1}$, where $H_{1}$ denotes the primary segment through the fixed point $p$ in $W$.

Given $I \subset H_{1}$, let $z_{0}$ be the critical point in $H_{1}, \mathcal{P}_{\left[z_{0}\right]}$ denotes the partition of $H_{1} \cap(-\delta, \delta)$ relative to $z_{0}$, and $I_{\mu_{0}}, I_{-\mu_{0}}$ are outmost interval of $\mathcal{P}_{\left[z_{0}\right]}$. Without loss of generality, we assume $I \not \supset I_{ \pm \mu_{0}}, I \not \supset I_{ \pm\left(\mu_{0}-1\right)}$. Otherwise, by Lemma 3.3 and 3.4, we have that $I$ contains the transversal homoclinic points.

Now we consider two cases.

(I) $I \cap(-\delta, \delta) \times R^{m-1}=\emptyset$. 
First, we prove that there exist $n_{1}$ and $\mu_{1}>0$ such that $\varphi^{n_{1}} I \supset I_{\mu_{1}}$ or $\varphi^{n_{1}} I \supset$ $I_{-\mu_{1}}$. If $\mu_{1}=\mu_{0}$ or $\mu_{1}=\mu_{0}-1$, then by Lemma 3.3 and 3.4, we have that $I$ contains the transversal homoclinic point.

From Lemma 2.1 and 2.2, it follows that there exists $n$ such that $\varphi^{n} I$ intersects $(-\delta, \delta) \times R^{m-1}$ and $\varphi^{n} I \cap(-\delta, \delta) \times R^{m-1}$ bound to some point $z_{1} \in \mathcal{C}$, moreover $\varphi^{n} I$ is almost horizontal.

If $\varphi^{n} I \cap(-\delta, \delta) \times R^{m-1}$ contains some $I_{\mu}$, then let $n_{1}=n$ and $\mu_{1}$ denote the minimal absolute value of $\mu$.

Otherwise we have two subcases:

(1) $\varphi^{n} I \cap(-\delta, \delta) \times R^{m-1} \subset I_{\mu_{0}} \times R^{m-1}$, moreover $\varphi^{n} I \cap\left[(-\delta, \delta) \times R^{m-1}\right]^{c} \neq \emptyset$. If case (1) occurs, moreover the segment $\varphi^{n} I \cap\left[(-\delta, \delta) \times R^{m-1}\right]^{c}$ is contained in $I_{\left(\mu_{0}-1\right)}$ or $I_{-\left(\mu_{0}-1\right)}$. Then keep iterating $\varphi^{n} I$. From Lemmas 2.1 and 2.2, there must be some $n^{\prime}$ such that $\varphi^{n^{\prime}+n} I$ intersects $(-\delta, \delta) \times R^{m-1}$ again. Moreover $\left|\varphi^{n^{\prime}+n} I\right|>2\left|\varphi^{n} I\right|$ and $\varphi^{n^{\prime}+n} I$ is almost horizontal.

If case (1) occurs, but the segment $\varphi^{n} I \cap\left[(-\delta, \delta) \times R^{m-1}\right]^{c}$ contains $I_{\left(\mu_{0}-1\right)}$ or $I_{-\left(\mu_{0}-1\right)}$, then let $n_{1}=n$ and $\mu_{1}=\mu_{0}-1$.

(2) $\varphi^{n} I \subset J_{\mu}^{+}$.

If this case occurs, then keep iterating $\varphi^{n} I$. From Lemma 2.1 (II) and Remark 1, it follows that $\left|\varphi^{n+p} I\right| \geq e^{\frac{1}{6} c_{1} p}\left|\varphi^{n} I\right|>2\left|\varphi^{n} I\right|$. Keep iterating $\varphi^{n+p} I$ until there is some $n^{\prime \prime}$ such that $\varphi^{n+p+n^{\prime \prime}} I \cap(-\delta, \delta) \times R^{m-1}$ is nonempty. By Lemma 2.1, we have $\left|\varphi^{n+p+n^{\prime \prime}} I\right|>\left|\varphi^{n+p} I\right|>2\left|\varphi^{n} I\right|$, and by Lemma 2.2 , we have $\varphi^{n+p+n^{\prime \prime}} I$ is almost horizontal.

By repeating the above arguments, we conclude that there must be $n_{1}$ such that $\varphi^{n_{1}} I$ contain some $I_{\mu}$ and $\varphi^{n_{1}} I$ is almost horizontal, denote the minimal absolute value of $\mu$ by $\mu_{1}$.

Next, we iterate $I_{\mu_{1}}$ or $I_{-\mu_{1}}$ (in term of $\varphi^{n_{1}} I \supset I_{\mu_{1}}$ or $I_{-\mu_{1}}$ ). By Lemma 2.1 (II), it follows that

$$
\left|\varphi^{p\left( \pm \mu_{1}\right)} I_{ \pm \mu_{1}}\right| \geq e^{\frac{1}{3} c_{1} p\left( \pm \mu_{1}\right)}\left|I_{ \pm \mu_{1}}\right| .
$$

There exists $l\left( \pm \mu_{1}\right) \geq 0$ such that $\varphi^{l\left( \pm \mu_{1}\right)+p\left( \pm \mu_{1}\right)} I_{ \pm \mu_{1}}$ intersects $(-\delta, \delta) \times R^{m-1}$. If $\varphi^{l\left( \pm \mu_{1}\right)+p\left( \pm \mu_{1}\right)} I_{ \pm \mu_{1}}$ contains $I_{\mu}$ of some $\mathcal{P}_{[z]}$, then denote the minimal absolute value of $\mu$ by $\mu_{2}$. Otherwise repeat the arguments as above, keep iterating; there must be $n_{2}$ such that $\varphi^{n_{2}} I_{ \pm \mu_{1}}$ contains some $I_{\mu}$ and $\varphi^{n_{2}} I_{ \pm \mu_{1}}$ is almost horizontal, then denote the minimal absolute value of $\mu$ by $\mu_{2}$.

From Lemma 2.1, it follows that $\left|\varphi^{n_{2}} I_{\mu_{1}}\right| \geq e^{\frac{1}{3} c_{1} p}\left|I_{\mu_{1}}\right|$, since $p \geq \frac{1}{2} \mu_{1}$, it has $\left|\varphi^{n_{2}} I_{\mu_{1}}\right| \geq 2 e^{-\left(\mu_{1}-1\right)}$. Thus we get that $0<\mu_{2}<\mu_{1}$.

We then consider $I_{\mu_{2}}$ or $I_{-\mu_{2}}$ as above, repeat the arguments as above, and we have $0<\mu_{k}<\mu_{k-1}<\cdots<\mu_{2}<\mu_{1}$. Therefore there must be $\tilde{N}$, such that $\varphi^{\tilde{N}} I$ contains an almost horizontal segment $J$ which contains some $I_{i}$, for $i= \pm \mu_{0}$, $\pm\left(\mu_{0}-1\right)$.

From Lemma 3.4, for $b_{0}$ small enough, if $b<b_{0}$, then $\varphi^{N} \varphi^{\tilde{N}} I$ or $\varphi^{M} \varphi^{\tilde{N}}$ intersects $w^{s}(p)$ transversally. Hence $I$ contains the transversal homoclinic points.

(II) $I \cap(-\delta, \delta) \times R^{m-1} \neq \emptyset$.

If $I$ contains some $I_{\mu}$, let $\mu_{1}$ denote the minimal absolute value of $\mu$. Keep iterating, analogous to (I). If not, then $I$ must be contained in some $J_{\mu}^{+}$for $\mu \geq \mu_{0}$, keep iterating as above. We also have that $I$ contains the transversal homoclinic points. 
Summarizing (I) and (II), it follows that the transversal homoclinic points are dense in $H_{1}$. Therefore the transversal homoclinic points are dense in $W$.

Corollary. The hyperbolic periodic points are dense in the codimension-1 Hénonlike strange attractor. Moreover the hyperbolic periodic points that are heteroclinically related to the primary fixed point are also dense in it.

Proof. From the proof of the Theorem, it follows the transversal homoclinic points of $w^{s}(p)$ are dense in $\Lambda$. By the Smale-Birkhoff Theorem, we have that the hyperbolic points that are heteroclinically related to the primary fixed point $p$ are dense and the hyperbolic periodic points are dense in $\Lambda$.

\section{ACKNOWLEDGMENTS}

Part of the work was done while the author visited IMPA. The author thanks TWAS, IMPA for their support and hospitality, Professor M. Viana and Zhang Zhifen for their guidance and encouragement, Professor L. S. Young and J. Kennedy for their discussions, and the referees for their suggestions.

\section{REFERENCES}

1. M.Benedicks \& L.Carleson, The dynamics of the Hénon map, Ann. Math. 133 (1991), 73-169. MR 92d:58116

2. M.Benedicks \& L.S.Young, Sinai-Bowen-Ruelle measures for certain Hénon maps, Inventions Math. 112 (1993), 541-576. MR 94e:58074

3. M.Hénon, A two-dimensional mapping with a strange attractor, Commun. Math. Phys 50 (1976), 69-77. MR 54:10917

4. L.Mora \& M.Viana, Abundance of strange attractors, Acta .Math. 171 (1993), 1-71. MR 94k:58089

5. J.Palis \& F.Takens, Hyperbolicity and sensitive chaotic dynamics at homoclinic bifurcations, Cambridge Press, 1993. MR 94h:58129

6. M.Viana, Strange attractors in higher dimensions, Bull.Braz.Math.Soc. 24 (1993), 13-62. MR 94k:58093

Department of Mathematics, Suzhou University, Suzhou 215006, Jiangsu, People's Republic of China

E-mail address: ylcao@nsad.suda.edu.cn 